\title{
Analysis of percutaneous kyphoplasty or short-segmental fixation combined with vertebroplasty in the treatment of Kummell disease
}

Wei Lu' ${ }^{1}$, Long Wang ${ }^{1,2}$, Chunlin Xie ${ }^{3}$, Zhaowei Teng ${ }^{4}$, Gonghai Han ${ }^{5,6}$, Rongmao Shi ${ }^{1}$, Jinlong Liang ${ }^{1}$ and Sheng $L^{1,5,6^{*}}$

\begin{abstract}
Background: In recent years, short segment internal fixation combined with vertebroplasty (SSF + VP) has provided a new option for the treatment of Kummell disease (KD). The purpose of this study is to evaluate the efficacy of percutaneous kyphoplasty (PKP) and SSF + VP, to provide evidence-based medical support for the decision-making process when treating KD patients without neurological deficits.

Methods: Databases including MEDLINE (PubMed) and EMBASE (Ovid) (1947 to April 6, 2019) were searched for PKP and short-segmental fixation combined with vertebroplasty (SSF + VP) to treat Kummell disease in randomized controlled trials (RCTs) or cohort studies. Two investigators independently evaluated the eligibility of the studies retrieved from the databases based on the predetermined selection criteria. The postoperative evaluation indexes included operation time, bleeding volume, visual analog scale (VAS) score, Oswestry Disability Index (ODI) score, local vertebral Cobb angle, and cement leakage. When the data were significant, a random-effects model was used for analysis. In contrast, when the results showed no statistical heterogeneity, a fixed-effects model was used to estimate the overall effect sizes.
\end{abstract}

Results: Three retrospective case-control studies were included in the final analysis. The differences in the bleeding volume and operation time were statistically significant, and the combined weighted mean differences (WMDs) $(95 \% \mathrm{Cl})$ were $-0.204 .46(-210.97,-197.93)$ and $-98.98(-141.63,56.32)$, respectively.

The combined data showed that the differences in VAS score, ODI score, local vertebral Cobb angle, and cement leakage were not statistically significant.

Conclusions: This analysis demonstrates that the PKP and SSF + VP methods are safe and effective in treating Kummell disease patients without neurological symptoms. However, PKP can shorten the operation time and reduce the volume of blood loss.

Keywords: Kyphoplasty, Vertebroplasty, Fixation, Kummell disease

\footnotetext{
* Correspondence: drlusheng@163.com

${ }^{1}$ Clinical College of 920th Hospital of Joint Logistics Support Force of

Kunming Medical University, No. 212 Daguan Road, Kunming 65032, Yunnan,

People's Republic of China

${ }^{5}$ Department of Orthopaedics, The First Peoples' Hospital of Yunnan

Province, Kunming 650032, Yunnan, People's Republic of China

Full list of author information is available at the end of the article
}

(C) The Author(s). 2019 Open Access This article is distributed under the terms of the Creative Commons Attribution 4.0 International License (http://creativecommons.org/licenses/by/4.0/), which permits unrestricted use, distribution, and

reproduction in any medium, provided you give appropriate credit to the original author(s) and the source, provide a link to the Creative Commons license, and indicate if changes were made. The Creative Commons Public Domain Dedication waiver (http://creativecommons.org/publicdomain/zero/1.0/) applies to the data made available in this article, unless otherwise stated. 


\section{Background}

Kummell disease (KD) was first reported by Steel in 1951 and occurs in middle-aged and elderly people with osteoporosis. KD presents as a delayed vertebral compression fracture and is characterized by the following common characteristics: a history of minor trauma, after which the pain disappears, but the symptoms recur or worsen, and a kyphosis deformity occurs months or years later $[1,2]$. The affected vertebra is usually located in the lower thoracic or upper lumbar region (T8-L4), owing to the well-known prevalence of vertebral fractures at the thoracolumbar junction. In the majority of cases, only a single vertebra is involved [2].

The main diagnostic imaging finding of KD is characterized by an intravertebral vacuum cleft on plain radiograph, which is better appreciated on the anteroposterior view of computed tomography (CT) or magnetic resonance imaging (MRI) scans [3]. However, imaging cannot be used as a specific basis for diagnosis. KD is surrounded by hardened bone and cannot self-heal. At present, there is no standard treatment for KD $[1,4]$.

In terms of surgical treatments, different methods are adopted according to the presence of neurological symptoms. If the patients are neurologically impaired, the aim of surgery is to decompress the spinal canal, restore the spinal curvature, and maintain spinal stability. The surgical modes include anterior, posterior, or combined anterior and posterior approaches. If the patients do not have neurological symptoms, the purpose of surgery is to preserve the maximum amount of movement of the injured vertebra and to restore vertebral height and sagittal alignment. Therefore, the percutaneous kyphoplasty (PKP) and percutaneous vertebroplasty (PVP) techniques have been widely used to treat KD [5, 6]. However, there have been reports of loosening and displacement of the bone cement, further loss of vertebral height, and even secondary paralysis following PKP or PVP [7-10]. Therefore, short-segmental fixation combined with vertebroplasty (SSF + VP) has also been used to treat KD in recent years. This technique has been reported to have certain positive effects on pain relief and functional recovery $[1,11,12]$.

However, no consensus has been reached on the optimal treatment method for KD patients without neurological deficits. Thus, we performed an analysis to evaluate the efficacy of PKP and SSF + VP and to provide evidencebased medical support for the decision-making process when treating KD patients without neurological deficits.

\section{Methods}

\section{Search strategy and data sources}

We searched MEDLINE (PubMed) and EMBASE (OVID) (1947 to April 6, 2019) for randomized controlled trials (RCTs) or cohort studies that investigated PKP and SSF + VP to treat KD. There were no restrictions regarding language or type of publication. The search terms used were the following: (i) kummell [Title/Abstract]) OR avascular osteonecrosis of vertebral body [Title/Abstract]) OR vertebral osteonecrosis [Title/ Abstract]) OR vertebral pseudarthrosis [Title/Abstract]) OR intravertebral vacuum cleft [Title/Abstract]) OR delayed vertebral collapse [Title/Abstract]) OR compression fracture nonunion [Title/Abstract]; AND (ii) kyphoplasty [Title/Abstract]) OR vertebroplasty [Title/Abstract]) OR bone cement augment [Title/Abstract]) OR fixation [Title/ Abstract]. The retrieval strategy was formulated according to a professional retrieval process, and we also searched the bibliographies of relevant articles to identify any additional studies.

\section{Study selection}

The inclusion criteria were as follows: (1) presented original data from a cohort study or case-control study; (2) included patients definitively diagnosed with KD; (3) used two comparator groups in which one group was treated with a PKP strategy, and the other group was treated with an SSF + VP strategy; (4) included patients with monosegmental lesions who did not have neurological deficits and for whom conservative treatment was invalid; and (5) had sufficient data for analysis.

The exclusion criteria were as follows: (1) included patients with metastatic spinal tumors, infections, primary bone tumors, multiple myeloma or bisegmental and multisegmental lesions; (2) included patients with neurological symptoms; (3) included patients with defects of the posterior wall of the vertebral body or those that occupied the vertebral canal; and (4) included non-human study subjects. If the data were duplicated or the same population was used in more than one study, we chose the most recent or complete study.

\section{Data extraction and quality assessment}

Two investigators (Wei Lu/Zhaowei Teng) independently evaluated the eligibility of the studies retrieved from the databases based on the predetermined selection criteria. In addition, a cross-reference search for eligible articles was conducted to identify studies not identified from the computerized search. These two authors independently extracted the following data: the first author's name; year of publication, study regions, cohort size, operative time, bleeding volume, visual analog scale (VAS) score, Oswestry Disability Index (ODI) score, local vertebral Cobb angle, cement leakage, and statistical adjustments for confounding factors. Any disagreements were resolved either by discussion or in consultation with the corresponding author (Sheng Lu). Finally, the eligible studies were included in the meta-analysis. 


\section{Statistical analyses}

Data analysis was performed using Stata 14.0 software (StataCorp., USA). The Cochran $Q$ and $I^{2}$ value were used together to test heterogeneity. When the $p$ value was $<0.1$ and the $I^{2}$ value was $>50 \%$, the data were considered to be heterogeneous, and a random-effects model was used for the meta-analysis. Otherwise, when the results showed no statistical heterogeneity, a fixedeffects model was used to estimate the overall effect sizes.

\section{Results}

\section{Literature search and study characteristics}

A total of 329 articles were initially identified from the PubMed and EMBASE databases. There were no additional studies from other sources. After removing the duplicate articles, 73 studies were included for further assessment. We reviewed the titles, abstracts, and full texts of all retrieved articles using the defined criteria. Finally, there were three articles that met the inclusion criteria. Figure 1 shows the flow diagram of the selection process. The characteristics of the included studies are shown in Table 1. All three articles were retrospective case-control studies from China [13-15].

\section{Analysis}

The data of the three included articles were summarized and analyzed. Two indicators obtained from the forest map were statistically significant, namely, the volume of blood loss during surgery and the operation time. Since the specific volume of blood loss was not provided in one paper (Chen, China) [14], we combined the data of the other two papers, as shown in Fig. 2a. The combined weighted mean difference (WMD) $(95 \% \mathrm{CI})$ was $0.204 .46(-210.97,-197.93)$, and the combined results were statistically significant.

The operation time data of the three references were combined. As shown in Fig. $2 \mathrm{~b}$, the combined WMD $(95 \% \mathrm{CI})$ was $-98.98(-141.63,-56.32)$, and the combined results were statistically significant.

Data from the three included studies regarding the VAS score, ODI score, and local vertebral Cobb angle of the

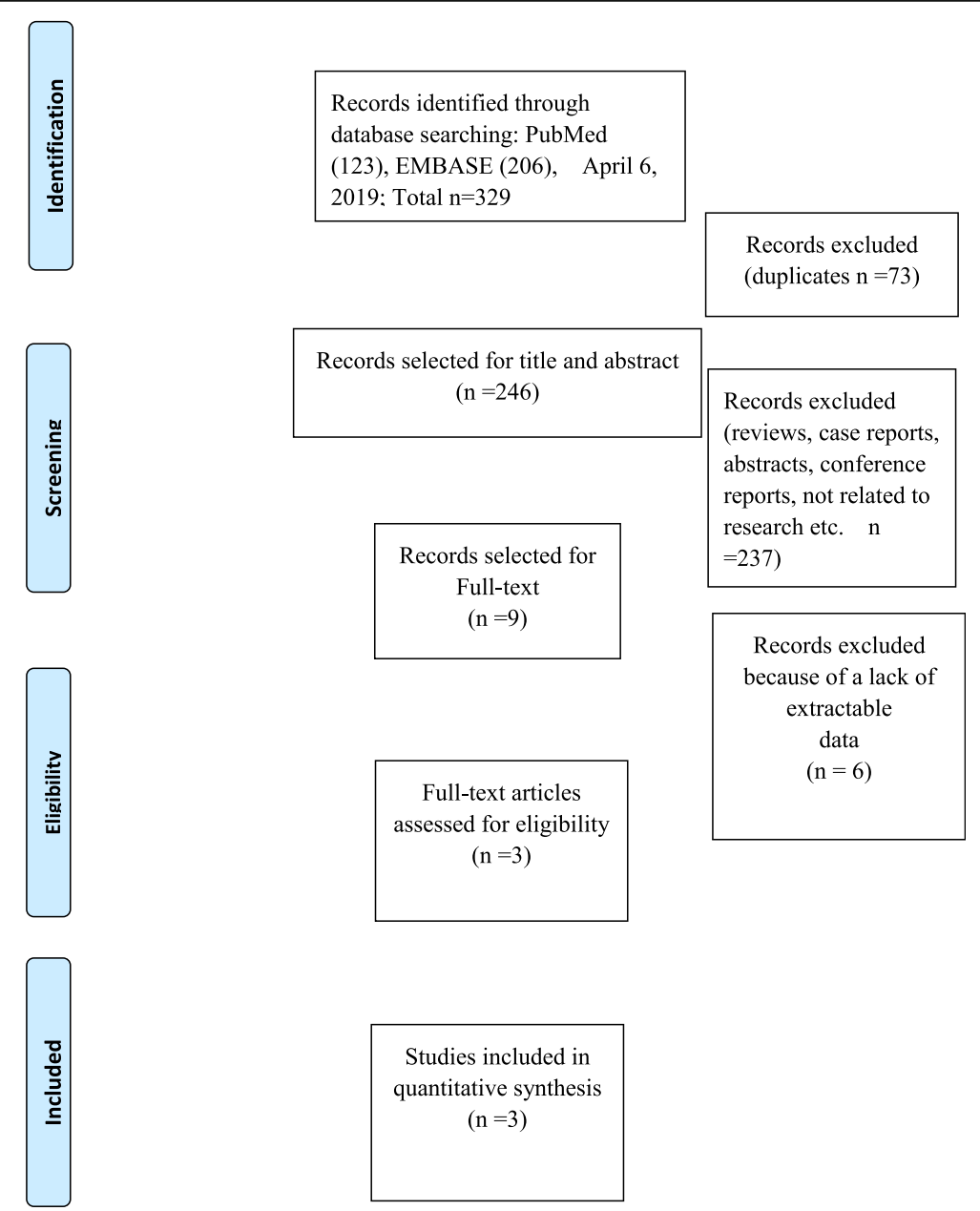

Fig. 1 Flow chart illustrating the literature search process in this study 


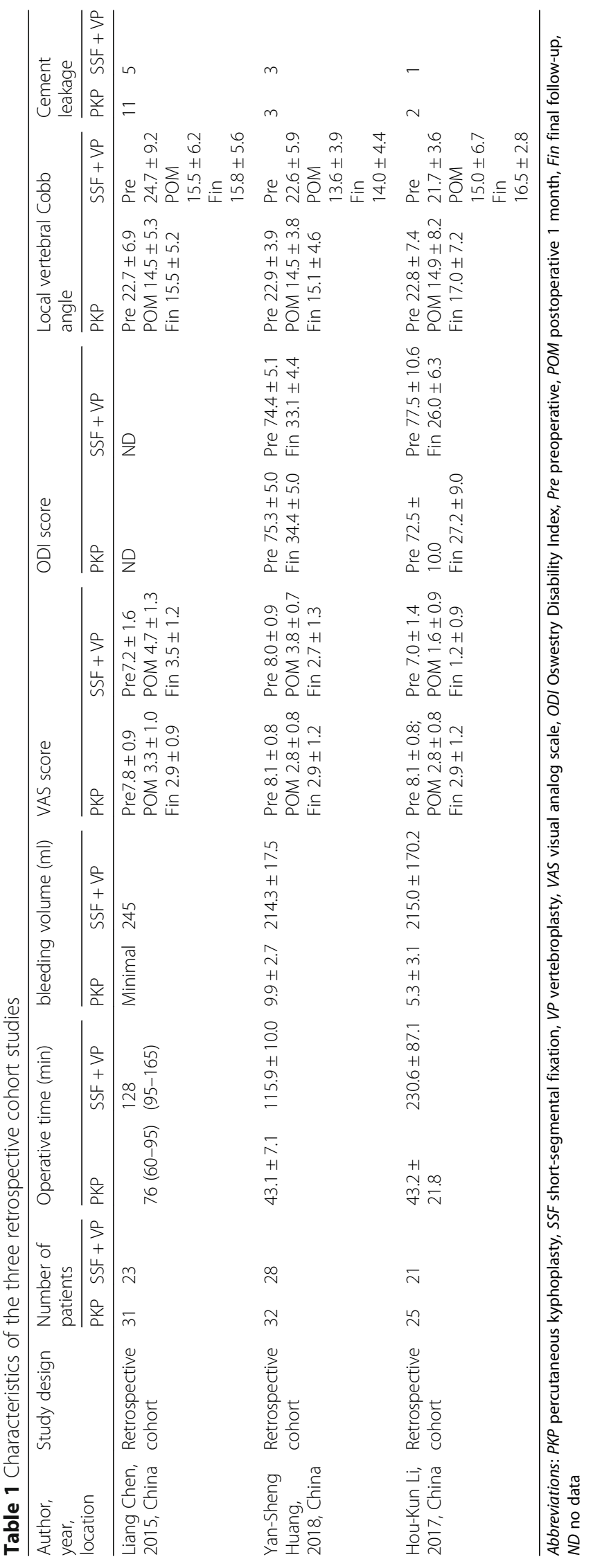




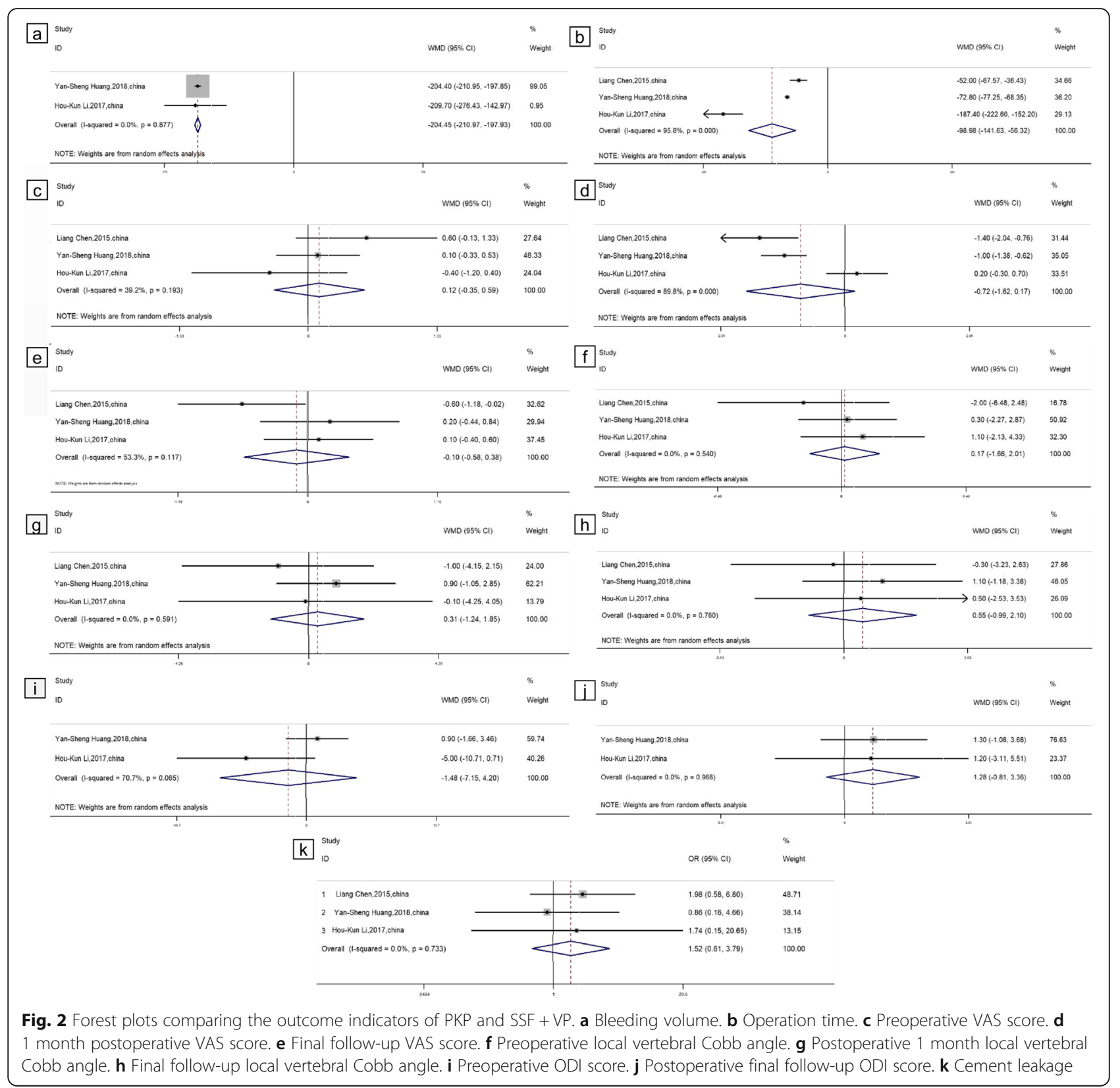

two different surgical methods PKP and SSF + VP were extracted and combined. We combined the VAS score data in the preoperative period, at 1 month postoperative and at the final follow-up. As shown in Fig. 2c-e, the combined WMDs $(95 \% \mathrm{CI})$ were $0.12(-0.35,0.58), 0.72$ $(-1.62,0.17)$, and $-0.10(-0.58,0.38)$ in the preoperative period, at 1 month postoperative and at the final followup, respectively. The combined data showed no statistical significance.

We combined the local vertebral Cobb angle data in the preoperative period, at 1 month postoperative, and at the final follow-up, as shown in Fig. $2 \mathrm{f}-\mathrm{h}$. The corresponding WMDs $(95 \% \mathrm{CI})$ were $0.17(-1.66,2.01), 0.31$
$(-1.24,1.85)$, and $0.55(-0.99,2.10)$; the combined data were not statistically significant.

Because (Chen, China) [14] did not provide ODI scores, the ODI scores in the preoperative period and at the postoperative final follow-up of the two remaining papers were combined, as shown in Fig. $2 \mathrm{i}, \mathrm{j}$. The WMDs $(95 \% \mathrm{CI})$ in the preoperative period and at the postoperative final follow-up were $-1.48(-7.25,4.20)$ and $1.28(-0.81,3.36)$, respectively; the combined data were not statistically significant.

The cement leakage data were combined, as shown in Fig. 2k, with an OR (95\% CI) value of $1.52(0.61,3.79)$; the combined data were not statistically significant. 


\section{Discussion}

In the past, KD was considered a rare disease. However, with the development of osteoporosis research and medical imaging technology, the diagnostic rate of osteoporotic vertebral fracture has increased in recent years. KD cannot be treated by conservative treatment such as lying in bed or with a brace [16]. KD is progressive and thus, self-healing is difficult. KD can cause long-term low back pain. If the fracture is not treated in time, the collapsed vertebral body can also compress the spinal cord and can even cause paralysis [2, 17, 18]. Compared with PVP, PKP has advantages in correcting kyphosis and reducing bone cement leakage. PKP has been widely used to treat KD patients without neurological symptoms. However, the special pathological changes of KD lead to poor dispersion of the bone cement through the cancellous bone, resulting in reduced mechanical stability. Cases of bone cement displacement, loss of vertebral body height, and even secondary paralysis have been reported in the literature [7-10]. Recently, short-segmental fixation combined with vertebroplasty $(\mathrm{SSF}+\mathrm{VP})$ has emerged as another option for $\mathrm{KD}$ treatment $[1,11,12]$.

To the best of our knowledge, this analysis is the first to evaluate the efficacy of these two methods and provide evidence-based medical support for the decision-making process when treating KD patients without neurological deficits. Our study included three retrospective case-control studies [13-15]. Because one paper (Chen, China) [14] did not provide ODI scores or bleeding volume data, we included only the ODI score and bleeding volume data from the remaining two articles $[13,15]$. The included operation time, VAS score, local vertebral Cobb angle, and cement leakage data were from all three studies [13-15].

According to our data analysis results, the combined data showed that the VAS score, ODI score, local vertebral body Cobb angle, and cement leakage were not significantly different between the two methods. The two surgical methods had a similar effect on improving the VAS score and ODI score and correcting the local vertebral body Cobb angle; the incidence of cement leakage was similar for both methods.

At present, the optimal treatment strategy is still controversial; however, the main purpose of both the PKP and SSF + VP surgical treatments is to relieve pain, restore vertebral height, and correct kyphosis. To improve treatment efficacy and patient satisfaction, the most important aspect is to relieve pain associated with KD. Pain is mainly due to the micromovements of the vertebral fracture. Therefore, eliminating the microfractures to achieve vertebral stabilization is very important. As a filler, bone cement provides immediate stability through microinterlock, volume-filling and bulk-filling mechanisms [19]. KD patients do not have obvious early symptoms, but these patients usually experience severe back pain and kyphosis by the time of diagnosis. Moreover, unlike common osteoporotic vertebral compression fractures (OVCFs), KD is characterized by sclerosis and bone resorption. Thus, the type of cement filling applied for treatment is very special. For most patients, bone cement is used as a support block because the bone cement does not diffuse sufficiently into the surrounding cancellous bone. Therefore, the anchoring effect between cement and bone is insufficient. In theory, SSF + VP will provide a more effective fixation than PKP and therefore will also lead to better pain relief, but this is not the case. The reason may be because PKP provides sufficient stability to the vertebra and destroys the surrounding pain sensory nerves $[19,20]$.

Bone cement leakage is a common complication and affects the patient's surgical safety outcomes and prognosis [21]. The incidence of bone cement leakage is significantly increased for KD patients [22-24].

Some studies $[11,12,20]$ believe that with the SSF + VP method, the bone cement can be injected under direct visualization after vertebral body reduction, which not only leads to a low incidence of bone cement leakage but also achieves a favorable vertebral height recovery. However, there were no significant differences in our study, most likely because the sample size was small.

In contrast, the combined data showed that the differences in bleeding volume and operation time were statistically significant. Relative to SSF + VP, PKP can shorten the surgical time and reduce the amount of bleeding. Moreover, Formica et al. [25] proposed a classification based on imaging findings that combines local and global sagittal parameters to help personalize the diagnosis and treatment of KD. Some studies [26, 27] suggest that vertebroplasty combined with posterior fixation can achieve a better sagittal deformity correction. KD usually occurs in adults over the age of 50, so the elderly patients in this study may have multiple comorbidities and severe osteoporosis; for such patients, the goals of the surgery should be to ensure safety and efficacy, minimize trauma, reduce bleeding, and shorten the operation time. These goals will maximize the benefits to the patient, thereby reducing the so-called domino effect of new fractures in adjacent segments due to poor sagittal alignment. Therefore, we believe that clinically, the decision to adopt SSF + VP should be made cautiously, and the decision requires a comprehensive analysis of each specific case.

In conclusion, both the PKP and SSF + VP methods are safe and effective for treating $K D$ patients without neurological symptoms. However, PKP can shorten the operation time and reduce the bleeding volume. However, due to the small number of randomized controlled studies included in this systematic evaluation, more prospective randomized controlled studies are needed to 
strengthen the credibility of this result. In addition, this study also has the following limitations: first, there was some statistical heterogeneity between the included studies. Although we used the random-effects model to balance this statistical heterogeneity in the analysis process, this heterogeneity still has some influence on the conclusions. Second, all the included studies were retrospective case-control studies, and this non-randomized controlled study was susceptible to selection bias, implementation bias, mixed bias, etc.

\section{Abbreviations}

CT: Computed tomography; Fin: Final follow-up; KD: Kummell disease; MRI: Magnetic resonance imaging; ND: No data; ODI: Oswestry Disability Index; PKP: Percutaneous kyphoplasty; POM: Postoperative 1 month; Pre: Preoperative; PVP: Percutaneous vertebroplasty; RCTs: Randomized controlled trials; SSF: Short-segmental fixation; VAS: Visual analog score; VP: Vertebroplasty; WMDs: Weighted mean differences

\section{Acknowledgements}

We appreciate the contribution of all patients, their families, the investigators, and the medical staff. We are grateful to all authors.

\section{Authors' contributions}

$W L$ and ZWT conceived the study design. WL, GHH, and CLX performed the data collection, data extraction, data interpretation, manuscript drafting, statistical analysis, and performance of the study. ZWT and WL were responsible for critical revision of the manuscript. $W L, L M, J L L, R M S$, and $H T L$ were responsible for data interpretation, manuscript drafting, supervision, and critical revision of the manuscript. SL and ZWT act as the guarantors for this article and take full responsibility for this study. All authors have read and approved the final manuscript.

\section{Funding}

This work was supported by the Major Science and Technology Special Project of Yunnan Province-Major Science and Technology Special Project (Biomedicine) [grant number: 2017ZF025].

\section{Availability of data and materials}

All data analyzed during this study are included in this published article.

\section{Ethics approval and consent to participate}

Not applicable.

\section{Consent for publication}

Not applicable

\section{Competing interests}

The authors declare that they have no competing interests.

\section{Author details}

${ }^{1}$ Clinical College of 920th Hospital of Joint Logistics Support Force of Kunming Medical University, No. 212 Daguan Road, Kunming 65032, Yunnan, People's Republic of China. 'Zhengzhou Orthopaedic Hospital, Zhengzhou 450052 HenanPeople's Republic of China. ${ }^{3}$ Department of Thoracic Surgery, The First Affiliated Hospital of Kunming Medical University, Kunming 650032, Yunnan, People's Republic of China. ${ }^{4}$ Department of Orthopedics, The Sixth Affiliated Hospital of Kunming Medical University, Yuxi 653100YunanPeople's Republic of China. ${ }^{5}$ Department of Orthopaedics, The First Peoples' Hospital of Yunnan Province, Kunming 650032, Yunnan, People's Republic of China. ${ }^{6}$ The Affiliated Hospital of Kunming University of Science and Technology, Kunming 650032, Yunnan, People's Republic of China.
Received: 7 June 2019 Accepted: 3 September 2019

Published online: 13 September 2019

\section{References}

1. Lee SH, Kim ES, Eoh W. Cement augmented anterior reconstruction with short posterior instrumentation: a less invasive surgical option for Kummell's disease with cord compression. J Clin Neurosci. 2011;18:509-14.

2. Li H, Liang CZ, Chen QX. Kümmell's disease, an uncommon and complicated spinal disorder: a review. J Int Med Res. 2012;40:406-14.

3. Malghem J, Maldague B, Labaisse MA, Dooms G, Duprez T, Devogelaer JP, et al. Intravertebral vacuum cleft: changes in content after supine positioning. Radiology. 1993;187:483-7.

4. Huilin $Y$, Jun $P$, Genlin W. A review of osteoporotic vertebral fracture nonunion management. Spine. 2014;39:B4-6.

5. Matsuura M, Fujimoto Y, Yamada K, Nakamae T. Percutaneous vertebroplasty versus balloon kyphoplasty for osteoporotic vertebral fracture with intravertebral cleft. Spine J. 2014;14:S123.

6. Huang $Y$, Peng M, He S, Tang X, Dai M, Tang C. Clinical efficacy of percutaneous kyphoplasty at the hyperextension position for the treatment of osteoporotic Kümmell disease. Clin Spine Surg. 2016;29:161-6.

7. Laredo JD. Expert's comment concerning grand rounds case entitled "Kümmell's disease: delayed post-traumatic osteonecrosis of the vertebral body" (by R. Ma, R. Chow, F. H. Shen). Eur Spine J. 2010;19:1071-2.

8. Wagner AL, Baskurt E. Refracture with cement extrusion following percutaneous vertebroplasty of a large interbody cleft. AJNR Am J Neuroradiol. 2006:27:230-1.

9. D'Oria S, Delvecchio C, Dibenedetto M, Zizza F, Somma C. Case report of Kummell's disease with delayed onset myelopathy and the literature review. Eur J Orthop Surg Traumatol. 2018;28:309-16.

10. Wang HS, Kim HS, Ju CL, Kim SW. Delayed bone cement displacement following balloon kyphoplasty. J Korean Neurosurg Soc. 2008;43:212-4.

11. Park SJ, Kim HS, Lee SK, Kim SW. Bone cement-augmented percutaneous short segment fixation: an effective treatment for Kummell's disease? J Korean Neurosurg Soc. 2015;58:54-9.

12. Zhang GQ, Gao YZ, Zheng J, Luo JP, Tang C, Chen SL, et al. Posterior decompression and short segmental pedicle screw fixation combined with vertebroplasty for Kümmell's disease with neurological deficits. Exp Ther Med. 2013;5:517-22.

13. Li HK, Hao DJ, Yang JS, Huang DG, Yu CC, Zhang JN, et al. Percutaneous kyphoplasty versus posterior spinal fixation with vertebroplasty for treatment of Kümmell disease. Medicine. 2017;96:e9287.

14. Chen L, Dong R, Gu Y, Feng Y. Comparison between balloon kyphoplasty and short segmental fixation combined with vertebroplasty in the treatment of Kummell's disease. Pain Physician. 2015;18:373-81.

15. Huang YS, Hao DJ, Feng H, Zhang HP, He SM, Ge CY, et al. Comparison of percutaneous kyphoplasty and bone cement-augmented short-segment pedicle screw fixation for management of Kümmell disease. Med Sci Monit. 2018;24:1072

16. Yoon ST, Qureshi AA, Heller JG. Kyphoplasty for salvage of a failed vertebroplasty in osteoporotic vertebral compression fractures: case report and surgical technique. J Spinal Disord Tech. 2005;18:S129-34.

17. Yu W, Liang YZ, Qiu T, Ye L, Jiang X. The therapeutic effect of intravertebral vacuum cleft with osteoporotic vertebral compression fractures: a systematic review and meta-analysis. Int J Surg. 2017:40:17-23.

18. Fabbriciani G, Pirro M, Floridi P, Callarelli L, Manfredelli MR, Scarponi AM, et al. Osteoanabolic therapy: a non-surgical option of treatment for Kummell's disease? Rheumatol Int. 2012;32:1371-4.

19. Garfin SR, Reilley MA. Minimally invasive treatment of osteoporotic vertebral body compression fractures. Spine J. 2002;2:76-80.

20. Kyriakou C, Molloy S, Vrionis F, Alberico R, Bastian L, Zonder JA, et al. The role of cement augmentation with percutaneous vertebroplasty and balloon kyphoplasty for the treatment of vertebral compression fractures in multiple myeloma: a consensus statement from the international myeloma working group (IMWG). Blood Cancer J. 2019;9:27.

21. Phillips FM, Wetzel FT, Lieberman I, Campbell-Hupp M. An in vivo comparison of the potential for extravertebral cement leak after vertebroplasty and kyphoplasty. Spine. 2002;27:2173-8.

22. Ha KY, Lee JS, Kim KW, Chon JS. Percutaneous vertebroplasty for vertebral compression fractures with and without intravertebral clefts. J Bone Joint Surg Br. 2006;88:629-33. 
23. Kong LD, Wang P, Wang LF, Shen Y, Shang ZK, Meng LC. Comparison of vertebroplasty and kyphoplasty in the treatment of osteoporotic vertebral compression fractures with intravertebral clefts. Eur J Orthop Surg Traumatol. 2014;24(Suppl 1):S201-8.

24. Tanigawa N, Kariya S, Komemushi A, Tokuda T, Nakatani M, Yagi R, et al. Cement leakage in percutaneous vertebroplasty for osteoporotic compression fractures with or without intravertebral clefts. AJR Am J Roentgenol. 2009;193:W442-5.

25. Formica M, Zanirato A, Cavagnaro L, Basso M, Divano S, Lamartina C, et al. Vertebral body osteonecrosis: proposal of a treatment-oriented classification system. Eur Spine J. 2018;27:190-7.

26. Huang YS, Hao DJ, Wang XD, Sun HH, Du JP, Yang JS, et al. Long-segment or bone cement-augmented short-segment fixation for Kummell disease with neurologic deficits? a comparative cohort study. World Neurosurg. 2018:116:e1079-86.

27. Nardi A, Tarantino U, Ventura L, Armotti P, Resmini G, Cozzi L, et al. Domino effect: mechanic factors role. Clin Cases Miner Bone Metab. 2011;8:38-42.

\section{Publisher's Note}

Springer Nature remains neutral with regard to jurisdictional claims in published maps and institutional affiliations.

Ready to submit your research? Choose BMC and benefit from:

- fast, convenient online submission

- thorough peer review by experienced researchers in your field

- rapid publication on acceptance

- support for research data, including large and complex data types

- gold Open Access which fosters wider collaboration and increased citations

- maximum visibility for your research: over $100 \mathrm{M}$ website views per year

At $\mathrm{BMC}$, research is always in progress.

Learn more biomedcentral.com/submissions 